\title{
APROVECHAMIENTO DE PELOS DE VACUNO DEL PROCESO DE PELAMBRE ENZIMÁTICO DE LAS CURTIEMBRES EN LA REMOCIÓN DE PLOMO
}

\author{
Carmen Carola Palomino Amorína*; Rocío Vargas Parkerb; Lizardo Visitación Figueroa ${ }^{\text {a }}$
}

\section{RESUMEN}

En este estudio, los residuos de pelos del proceso de pelambre enzimático (PPE) de una curtiembre fueron utilizados como adsorbentes para la remoción de plomo de una solución. Los residuos de pelos fueron tratados con hidrólisis ácida y utilizados en experimentos de adsorción tipo Batch en los cuales se evaluó el efecto del pH en la adsorción así como también el mecanismo de adsorción para lo cual se realizaron los estudios de las isotermas de adsorción y la cinética de adsorción de $\mathrm{Pb}$ (II) sobre los pelos tratados. Los resultados obtenidos mostraron que la adsorción de $\mathrm{Pb}$ (II) fue dependiente del $\mathrm{pH}$ siendo el pH óptimo de 7. Los estudios de la isoterma mostraron que los datos experimentales se ajustan con el modelo de la isoterma de Langmuir para los pelos tratados del proceso de pelambre enzimático (PTPE). La capacidad máxima de adsorción fue de 321,0 mg de $\mathrm{Pb}$ (II)/g de bioadsorbente (PTPE). Los estudios de cinética mostraron que el modelo de reacción de pseudo-segundo orden describe mejor el proceso de adsorción. Por último, el estudio mostró que los residuos de pelos del proceso de pelambre enzimático pueden ser utilizados como adsorbentes eficientes para la remoción de plomo (II).

Palabras clave: Pelo, plomo (II), adsorción, hidrólisis ácida, curtiembre.

\begin{abstract}
In this study, waste hairs of the enzymatic unhairing process (PPE) of tannery were used as adsorbents to remove lead of a solution. Waste hairs were treated with acid hydrolysis and used in the type batch adsorption experiments, to be examined the effect of $\mathrm{pH}$ on the adsorption and also to investigate the mechanism of adsorption for which studies adsorption isotherms and the adsorption kinetics of $\mathrm{Pb}$ (II) were performed on treated hairs. The results showed that adsorption of $\mathrm{Pb}$ (II) was $\mathrm{pH}$ dependent with the optimum $\mathrm{pH}$ of 7 . Isotherm studies showed that experimental data match the pattern of the Langmuir isotherm for the case of treated hairs of the enzymatic unhairing process (PTPE). The maximum adsorption capacity was $321.20 \mathrm{mg} \mathrm{Pb}$ (II)/g of bioadsobent (PTPE). The kinetic studies showed that

\footnotetext{
a* Centro de Investigación en Química, Toxicología y Biotecnología Ambiental del Departamento de Química de la Facultad de Ciencias de la Universidad Nacional Agraria La Molina. Lima-Perú. e-mail: carolapa16@gmail.com

${ }^{b}$ Helianthus SAC, Laboratorio de desarrollo e investigación curtiembre, Av. Guardia Civil 314 Chorrillos - LimaPerú.
} 
the reaction model of pseudo-second order best describes the adsorption process. Finally, the study showed that residues of hairs of the enzymatic unhairing process can be used as effective adsorbents for the removal of lead (II).

Key words: Hair, lead (II), adsorption, acid hydrolysis, tannery.

\section{INTRODUCCIÓN}

El pelambre en una curtiembre constituye una de las operaciones más contaminantes debido a la elevada carga de materia orgánica (proteínas, básicamente queratina y grasas suspendidas, así como compuestos tensoactivos usados como humectantes del cuero) e inorgánica como sulfuro, cal y otros en las aguas residuales ${ }^{1}$. Estas descargas en cuerpos de agua degradan la calidad de las mismas, ocasionando daños ambientales muchas veces irreversibles. Un cuerpo de agua contaminado por estas aguas residuales disminuye su valor de uso como agua para bebida o para fines agrícolas e industriales, afecta la vida acuática, genera mortandad de peces por disminución del oxígeno disuelto y el agua se convierte en no apta para el consumo.

Las formulaciones para la operación de pelambre varían, dependiendo del tipo de piel que se procese y del método de pelambre empleado. Los métodos de pelambre más comunes son: Pelambre de tipo convencional con destrucción de pelo, empleando sulfuro de sodio y cal y el pelambre con recuperación de pelo, que puede realizarse por diversos métodos, entre ellos el pelambre enzimático, que consiste en la combinación de enzimas (proteasas y queratinasas) con sulfuro, que remueven más eficientemente el pelo².

Pelos y plumas son subproductos residuales de industrias tales como las de curtido de pieles, las chacineras, los mataderos de pollos, etc. En la mayoría de los casos, estas biomasas, constituidas casi al $100 \%$ por la proteína más abundante en el planeta, es decir, la queratina, son incineradas o biodegradadas en vertederos sin llegar a ser utilizadas en diferentes procesos tecnológicos aplicados ${ }^{3}$.

Por tanto, el uso de adsorbentes a base de materiales orgánicos surge como una alternativa industrialmente atractiva como agentes de retención de metales debido a sus ventajas sobre otros tipos de tratamiento, como lo son: su capacidad de disminuir la concentración de iones metálicos a niveles de partes por billón ( $\mathrm{ppb}$ ), debido a su alta afinidad por los cationes metálicos, su fácil disponibilidad así como por su relativo bajo costo de elaboración ${ }^{4}$.

En particular, las proteínas de queratina son materiales potencialmente útiles para usar en los sistemas de adsorbentes o filtración para la eliminación de contaminantes tóxicos, debido al número de grupos funcionales presentes en las cadenas principales y secundarias de las proteínas, ${ }^{5}$ entre ellos tenemos a los grupos carboxilo (R-COOH), hidroxilo (R-OH), amino (R-NH2) y sulfhidrilo (R-SH). Entre los grupos funcionales, los átomos de azufre pueden coordinar más fácilmente con los iones de metales pesados. Por lo tanto, estos grupos funcionales permiten la fuerte atracción de los iones metálicos a las materias queratínicas, 
dándoles propiedades bioadsorbentes ideales para eliminación de metales pesados de las aguas residuales ${ }^{6}$.

Por otra parte, el plomo presente en aguas industriales de desecho constituye un problema ambiental de gran magnitud en razón de su elevada toxicidad para los organismos vivos. Las fuentes de contaminación por plomo para aguas naturales son diversas, siendo la principal la descarga de efluentes industriales directamente a fuentes naturales sin ningún tratamiento previo. Este problema se debe tanto a los altos costos como a la baja eficiencia de muchos de los métodos empleados para la remoción de los metales pesados.

A partir de lo mencionado anteriormente y como objetivo principal de este trabajo de investigación, se evaluó la posibilidad de utilizar los residuos de pelos de vacuno del proceso de pelambre enzimático como material adsorbente, capaz de remover el plomo de soluciones.

\section{PARTE EXPERIMENTAL}

\section{Materiales y métodos:}

\section{Material adsorbente de residuos de pelos:}

Se colectaron muestras de $2 \mathrm{~kg}$ de residuos de pelos de vacuno del proceso de pelambre enzimático de la industria de curtiembre. Estos residuos fueron donados por la empresa HELIANTHUS SAC, situada en el distrito de Chorrillos, departamento de Lima. Los residuos de pelo fueron lavados con $100 \%$ de agua a $28^{\circ} \mathrm{C}(2 \mathrm{~L}), 5 \%$ de sulfato de amonio $(0,1 \mathrm{~kg})$, $0,5 \%$ de Tandestal BASE $(0,01 \mathrm{~kg})$ y $1,0 \%$ de Tanplex FLA $(0,02 \mathrm{~kg})$ por un tiempo de 10 minutos con el fin de desencalarlos y disminuir el $\mathrm{pH} 12$ hasta 7 . Luego se lavaron con agua, se escurrieron y finalmente se secaron a temperatura ambiente.

\section{Hidrólisis ácida de la queratina de pelos de vacuno y caracterización}

La hidrólisis ácida de las muestras de pelos se realizó con $\mathrm{HCl} 8 \mathrm{M}(15,1 \mathrm{~mL} / \mathrm{g}$ pelo) durante $16,5 \mathrm{~h}$, luego fueron lavados con agua destilada, secados a temperatura ambiente, tamizados y homogenizados3. Los pelos fueron analizados después del tratamiento de hidrólisis ácida, por espectrofotometría infrarroja con transformada de Fourier (FTIR) en el equipo SHIMADZU IR Prestige-21.

\section{Determinación del plomo:}

La determinación de la concentración de $\mathrm{Pb}$ (II) en las soluciones filtradas se realizó utilizando como referencia el método 3310 B (espectrofotometría de adsorción atómica de flama) del Standard Methods (1998).

\section{Efecto del pH en la adsorción:}

Se realizaron mezclas de $50 \mathrm{ml}$ de una solución de $1,0 \mathrm{mg} / \mathrm{L}$ de $\mathrm{Pb}(\mathrm{II})$ con $0,1 \mathrm{~g}$ de residuos de pelos tratados del pelambre enzimático (PTPE), se les ajustó el pH a 3,0; 4,0; 5,0; 6,0; 7,0 y 8,0 y se agitaron durante 4 horas. Luego se filtraron, utilizándose la concentración de 
plomo de las soluciones resultantes para determinar la capacidad de bioadsorción de plomo en función del $\mathrm{pH}^{7}$. La cantidad de iones adsorbidos en el equilibrio fue calculada con la ecuación ${ }^{9}$ :

$$
q_{e(m g / L)}=\frac{\left(C_{i}-C_{e}\right) V}{W}
$$

y el porcentaje de remoción fue calculado con la ecuación:

$$
R(\%)=\frac{C_{i}-C_{e}}{C_{i}} \times 100
$$

Donde qe es la cantidad de adsorción en el equilibrio del metal $(\mathrm{mg} / \mathrm{g}), \mathrm{V}$ es el volumen de la solución muestra (L), W es el peso del adsorbente (g) y Ci y Ce son las concentraciones del metal en la solución acuosa (mg/L) antes y después del proceso de adsorción, respectivamente.

\section{Isotermas de adsorción}

Se mezclaron $50 \mathrm{~mL}$ de soluciones de 25, 50, 125, 250, 500, 700 y $1000 \mathrm{mg} / \mathrm{L}$ de plomo (II) con $0,1 \mathrm{~g}$ de PTPE (adsorbente), se les ajustó a $\mathrm{pH}=7$ y se agitaron durante 30 minutos. Posteriormente se determinó el contenido de plomo en las soluciones filtradas por espectrofotometría de absorción atómica para evaluar la capacidad máxima de adsorción (qmáx o Qm) de plomo7. Los resultados experimentales fueron correlacionados con los siguientes modelos de isotermas:

Para la forma lineal de la isoterma de Langmuir, la ecuación usada fue:

$$
\frac{C_{e}}{q_{e}}=\frac{1}{Q_{m} k_{a}}+\frac{1}{Q_{m}} * C_{e}
$$

Donde: $\mathrm{Q}_{\mathrm{m}}=$ Tasa máxima de adsorción del adsorbato ( $\mathrm{mg}$ de adsorbato/g biosorbente), $\mathrm{k}_{\mathrm{a}}=$ constante relacionada con la afinidad del biosorbente por el adsorbato.

Una gráfica de $C_{e} / q_{e}$ versus $\mathrm{C}_{\mathrm{e}}$ debe indicar una línea recta de pendiente $1 / \mathrm{Q}_{\mathrm{m}}$ y un intercepto de $1 /\left(Q_{m}{ }^{*} k_{a}\right)$.

Para la forma lineal de la isoterma de Freundlich, la ecuación utilizada fue la siguiente:

$$
\log q=\frac{1}{n} \log C_{e}+\log K
$$

Donde: $\mathrm{q}=$ concentración de soluto adsorbido por unidad de peso de adsorbente $(\mathrm{mg} / \mathrm{g})$. $\mathrm{Ce}=$ es la concentración del soluto en equilibrio $(\mathrm{mg} / \mathrm{L}) . \mathrm{n}=$ Constante relacionada con la intensidad de sorción del biosorbente en función de su grado de heterogeneidad. $\mathrm{K}=$ Constante de Freundlich relacionada a la capacidad de sorción del biosorbente. 


\section{Cinética de adsorción}

Se realizaron mezclas de $15 \mathrm{~mL}$ de una solución de $3000 \mathrm{mg} / \mathrm{L} \mathrm{Pb}$ (II) con $0,1 \mathrm{~g}$ de PTPE, se ajustaron a $\mathrm{pH}=7$ y se agitaron por periodos determinados $(0,5,10,15,30,60,90,120$, 240,360 y 480 minutos). Luego se analizó el contenido de plomo en las soluciones filtradas 4 . Los resultados experimentales fueron correlacionados con los siguientes modelos cinéticos:

La ecuación linealizada del modelo cinético pseudo-primer orden:

$$
q_{e(m g / L)}=\frac{\left(C_{i}-C_{e}\right) V}{W}
$$

Donde: $\mathrm{q}_{\mathrm{e}} \mathrm{y} \mathrm{q}_{\mathrm{t}}=$ son las capacidades de adsorción ( $\mathrm{mg} / \mathrm{g}$ ) en el equilibrio y en el momento $\mathrm{t}$, respectivamente. $\mathrm{k} 1=$ es la constante de adsorción de pseudo-primer orden $(\mathrm{L} / \mathrm{min})$.

La ecuación linealizada del modelo cinético de pseudo-segundo orden:

$$
R(\%)=\frac{C_{i}-C_{e}}{C_{i}} \times 100
$$

La capacidad de adsorción en el equilibrio y en el tiempo $t$ son representados por $\mathrm{q}_{\mathrm{e}} \mathrm{y}$ qt (mgg-1), respectivamente y k2 es la constante de adsorción de pseudo-segundo orden ( $\mathrm{g} /$ $(\mathrm{mg} \cdot \mathrm{min}))$.

\section{RESULTADOS Y DISCUSIÓN}

En la figura 1 se representa el espectro infrarrojo de los PTPE, en la cual se observan las bandas de adsorción a $3271.27 \mathrm{~cm}^{-1}$ atribuida al $\mathrm{NH}$ extendido, las bandas características de la queratina en los picos a 1631.78 (Amida I), 1519.91 (Amida II) y $1238.30 \mathrm{~cm}^{-1}$ (Amida III) y los productos de oxidación de la cistina en las bandas de adsorción a $1072.42 \mathrm{~cm}^{-}$ ${ }^{1}$ (monóxido de cistina) y a $1200-1150 \mathrm{~cm}^{-1}$ (sulfonatos). Asimismo, se observa un pico a $1049.28 \mathrm{~cm}^{-1}$ que representa a los ácidos sulfónicos, producto de la oxidación de los grupos tiol. Estos grupos se forman cuando el enlace disulfuro de la cistina se rompe por acción del sulfuro de sodio utilizado en el proceso de pelambre. Estos grupos son muy reactivos y se oxidan fácilmente generando productos con diversos estados de oxidación, como por ejemplo el ácido sulfónico, sulfonatos, etc. Los pelos tienen varios grupos funcionales (hidroxilo, amino, carboxilo y grupos conteniendo azufre como sulfonatos ${ }^{6}$ ) que tienen gran potencial de unión de metales, sin embargo, los principales grupos ionizables de unión catiónica en los biopolímeros son los carboxilos, fosfatos y sulfatos orgánicos ${ }^{8}$. Las especies químicas de plomo muestran una alta afinidad hacia grupos que contienen azufre?

En la figura 2 se representa el efecto del pH en la adsorción, este estudio se realizó en un rango de $\mathrm{pH}$ de 3 a 8 en condiciones constantes, en el cual se determinó que el $\mathrm{pH}$ en el que se produce la mayor remoción de $\mathrm{Pb}$ (II) por los residuos de pelos tratados del proceso de pelambre enzimático de la curtiembre (PTPE) está en el rango de 5 a 7 , considerándose el pH 7 como óptimo. 
Asimismo, se observa en esta figura que con el aumento en el $\mathrm{pH}$ de 3 a 5, el porcentaje de remoción de $\mathrm{Pb}$ (II) aumentó hasta llegar a un rango de $\mathrm{pH}$ de 5 a 7 en donde el porcentaje de remoción se mantiene relativamente constante, dándose en estos valores la mayor remoción. Sin embargo, con un incremento de $\mathrm{pH}$ mayor a 7 el porcentaje de remoción disminuye.

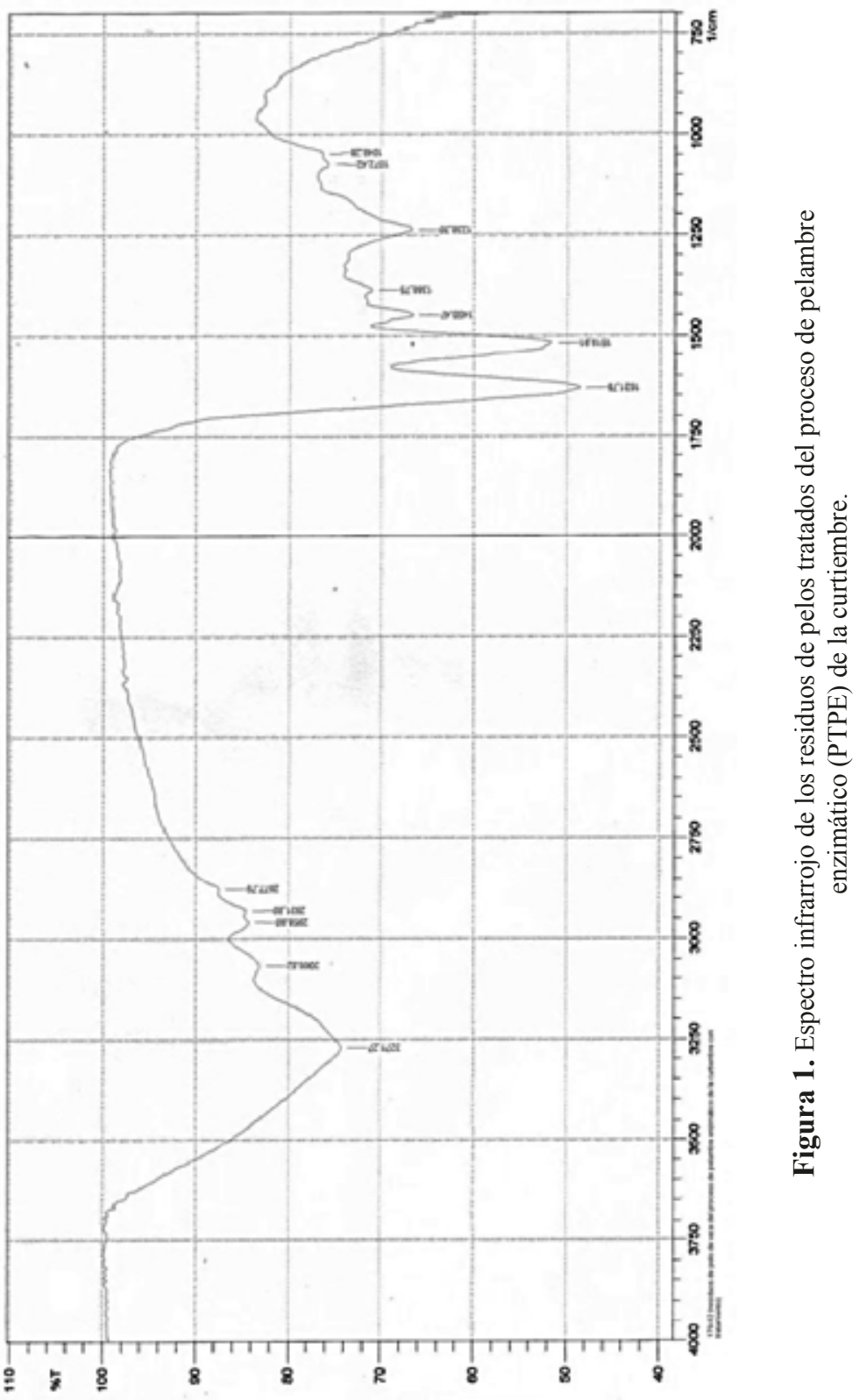




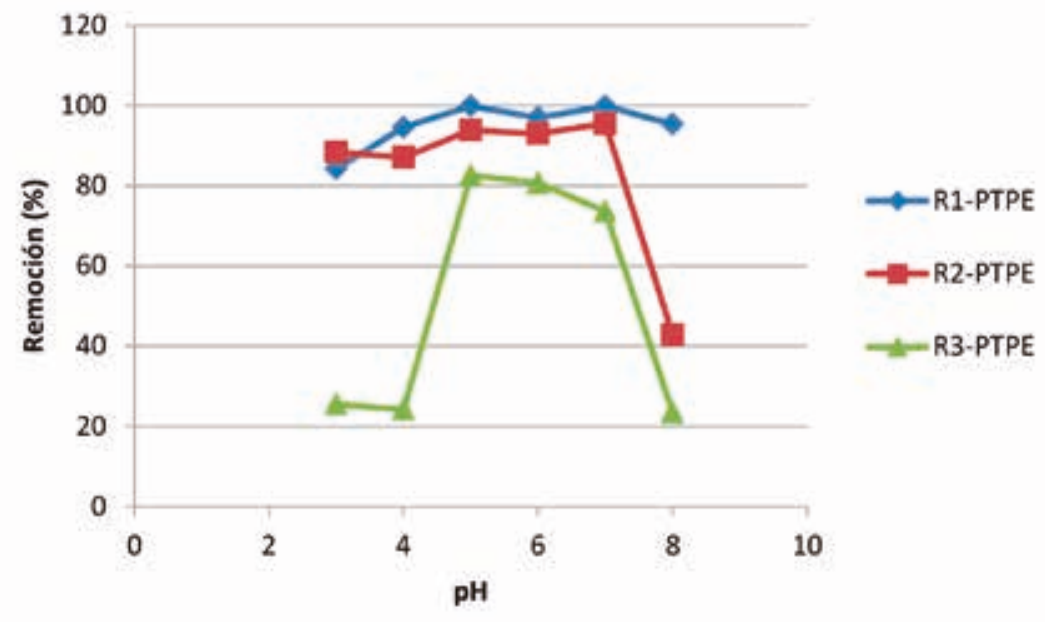

Figura 2. Efecto del pH en la adsorción del plomo (II) sobre residuos de pelo tratados del pelambre enzimático (PTPE) para tres repeticiones (R1, R2. R3).

El hecho que en medios muy ácidos disminuya de forma importante el porcentaje de remoción de plomo, podría ser el resultado de la competencia entre protones y los iones de plomo por los lugares de sorción, con una aparente preponderancia de los iones $\mathrm{H}^{+}$quedando cargados positivamente, lo que limita la aproximación de los cationes del metal como la consecuencia de las fuerzas de repulsión ${ }^{10-12}$. A medida que se eleva el $\mathrm{pH}$ del medio, el porcentaje de remoción de $\mathrm{Pb}$ (II) aumenta esto puede ser explicado por qué la superficie adsorbente es desprotonada y cargada negativamente pudiendo producirse la biosorción de los cationes metálicos ${ }^{13}$. Por otra parte, en valores superiores a $\mathrm{pH} 8$, el $\mathrm{Pb}$ (II) en solución, empieza a precipitar $\mathrm{y}$, por tanto, existe menos plomo disponible para ser retenido por el biosorbente $^{7}$, lo cual es confirmado con el diagrama de Pourbaix del sistema $\mathrm{Pb}-\mathrm{O}-\mathrm{H}$, que muestra la formación de un precipitado de $\mathrm{Pb}(\mathrm{OH})_{2}$ a un $\mathrm{pH}>8$, con lo cual habría menos plomo disuelto en la solución en estas condiciones.

Además, la queratina se comporta como un material aniónico cuando el pH de la solución es mayor que su punto isoeléctrico de $4.5^{5}$, por tanto, el pH óptimo ( $\mathrm{pH} 7$ ) escogido en este estudio favoreció el comportamiento aniónico de la queratina lo que permitió la unión del $\mathrm{Pb}$ (II) a los residuos de pelos.

En el estudio de isotermas de adsorción, los datos obtenidos se ajustaron al modelo linealizado de Langmuir y Freundlich, estableciéndose que el modelo de isoterma de Langmuir para tres repeticiones $\left(\mathrm{r}^{2}\right.$ promedio $=0,98$, ver tabla 1$)$ describe una mejor isoterma que el modelo 
de Freundlich $\left(\mathrm{r}^{2}\right.$ promedio $=0,78$, ver tabla 1$)$, debido a que tiene un mayor coeficiente de correlación, es decir, la ecuación linealizada del modelo representa de forma más exacta los resultados experimentales obtenidos. Esto sugiere que la adsorción de Pb (II) en los PTPE se produce a través de una adsorción monocapa sobre la superficie del adsorbente. Asimismo, indica que los grupos funcionales que se encuentran en la superficie del adsorbente actúan como sitios de adsorción de $\mathrm{Pb}$ (II) y adsorben el $\mathrm{Pb}$ (II) uno sobre uno ${ }^{14}$.

En la figura 3 a y b se observan los modelos linealizados de la isoterma de Langmuir y de la isoterma de Freundlich más representativos, obtenidos con los datos experimentales resultantes de la adsorción de $\mathrm{Pb}$ (II) sobre los residuos de pelos tratados, respectivamente. Comparando, se puede observar cómo los datos experimentales se ajustan mejor a la isoterma de Langmuir.

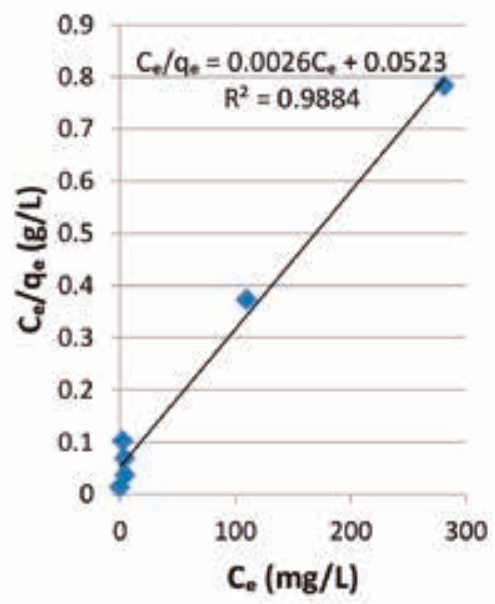

(a)

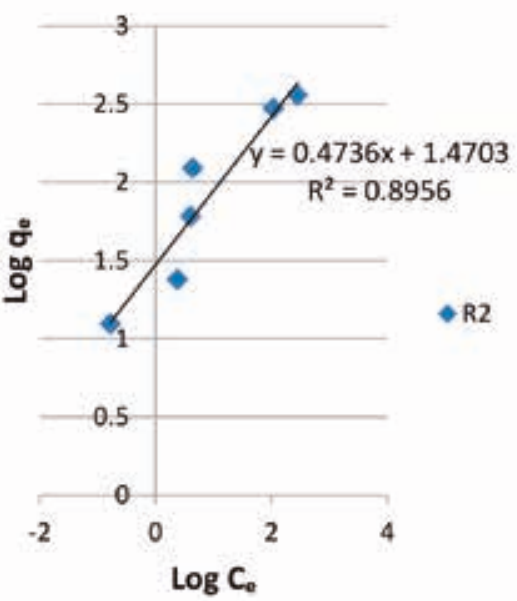

(b)

Figura 3. (a) Modelo linealizado de la isoterma de Langmuir de la adsorción de $\mathrm{Pb}$ (II) sobre PTPE (R2). (b) Modelo linealizado de la isoterma de Freundlich de la adsorción de Pb (II) sobre PTPE (R2). 
Así también, en la tabla 1 se presentan los parámetros obtenidos de los modelos linealizados de la isoterma de Langmuir y la isoterma de Freundlich de la adsorción de $\mathrm{Pb}$ (II) sobre PTPE.

Tabla 1. Parámetros de las isotermas obtenidas de la adsorción de Pb (II) sobre PTPE.

\begin{tabular}{ccccc}
\hline Muestra & $\mathrm{R} 1$ & $\mathrm{R} 2$ & $\mathrm{R} 3$ & Promedio \\
\hline Langmuir & & & & \\
\hline $\mathrm{Q}_{\mathrm{m}}(\mathrm{mg} / \mathrm{g})$ & 256,41 & 384,62 & 322,58 & 321,20 \\
$\mathrm{~K}_{\mathrm{a}}(\mathrm{L} / \mathrm{mg})$ & 0,0741 & 0,0497 & 0,0489 & 0,0576 \\
$\mathrm{R}^{2}$ & 0,97 & 0,99 & 0,98 & 0,98 \\
$\mathrm{~L}_{\mathrm{a}}$ & $2,54 \mathrm{E}-06$ & $1,69 \mathrm{E}-06$ & $1,69 \mathrm{E}-06$ & \\
\hline Freundlich & & & & \\
\hline $\mathrm{K}_{\mathrm{F}}(\mathrm{mg} / \mathrm{g})$ & 18,5823 & 29,5325 & 29,9985 & 26,0378 \\
$1 / \mathrm{n}$ & 0,6168 & 0,4736 & 0,4111 & 0,5005 \\
$\mathrm{R}^{2}$ & 0,76 & 0,90 & 0,69 & 0,78 \\
\hline
\end{tabular}

Se determinó mediante el modelo de la isoterma de Langmuir que la capacidad máxima de adsorción promedio de $\mathrm{Pb}$ (II) sobre PTPE, a pH óptimo, es de 321,20 mg de $\mathrm{Pb}$ (II)/g de PTPE (tabla 1).

Valores de capacidad máxima de adsorción para la isoterma de Langmuir de varios adsorbentes son reportados en la tabla 2. En el cual se puede observar que la capacidad máxima de adsorción de los PTPE es superior a varios adsorbentes y es comparable al del adsorbente de raquis de plátano ${ }^{15}$.

Tabla 2. Comparación de capacidades máximas de adsorción de otros adsorbentes de plomo (II).

\begin{tabular}{ccc}
\hline Adsorbente & $\begin{array}{c}\text { Capacidad máxima de } \\
\text { adsorción de } \mathrm{Pb}(\mathrm{II}) \\
(\mathrm{mg} / \mathrm{g})\end{array}$ & Referencia \\
\hline Partículas coloides de Keratina & 43,3 & $(14)$ \\
Carbón activado modificado & 29,44 & $(8)$ \\
Residuos de vegetales & 182 & $(5)$ \\
Cáscara de naranja pretratada & 141,05 & $(16)$ \\
Alpeorujo (residuos de olivar) & 25,247 & $(10)$ \\
Raquis de plátano & 370,7 & $(15)$ \\
PTPE & 321,2 & Este trabajo \\
\hline
\end{tabular}


En el estudio cinético de adsorción se observó que los datos experimentales se ajustan al modelo cinético de pseudo-segundo orden, dado que se obtiene un coeficiente de correlación promedio de $\mathrm{R}^{2}=0,98$, valor superior al obtenido por el modelo cinético de pseudo-primer orden de $\mathrm{R}^{2}=0,56$ (ver tabla 3 ). Es decir, explica mejor los datos cinéticos de adsorción de $\mathrm{Pb}$ (II) sobre los PTPE. Asimismo, en el modelo de pseudo-segundo orden de la tabla 3 se observa que el valor promedio de la capacidad de adsorción calculada con el modelo $\left(\mathrm{q}_{\mathrm{e} \text { (cal) }}=400\right)$ es similar al valor promedio de la capacidad de adsorción determinada experimentalmente $\left(\mathrm{q}_{\mathrm{e}(\exp )}=435,24\right)$; lo cual corrobora la afirmación anterior. Estos resultados sugieren que el paso limitante de la velocidad puede ser el proceso de adsorción química. En la quimisorción, los metales pesados se adhieren a la superficie del adsorbente por formación de un enlace químico (generalmente covalente) ${ }^{13,6}$, es decir implica un intercambio de electrones entre el adsorbato y la superficie del material adsorbente ${ }^{17,6}$. Este comportamiento concuerda con los resultados reportados por Zhang ${ }^{6}$.

Por tanto, se observa en la figura 4 a y b, el modelo cinético de pseudo-segundo orden y de pseudo-primer orden más representativos obtenidos con los datos experimentales resultantes de la adsorción de $\mathrm{Pb}$ (II) sobre PTPE, respectivamente. Comparando se puede observar que el modelo de pseudo-segundo orden describe mejor el proceso de adsorción.

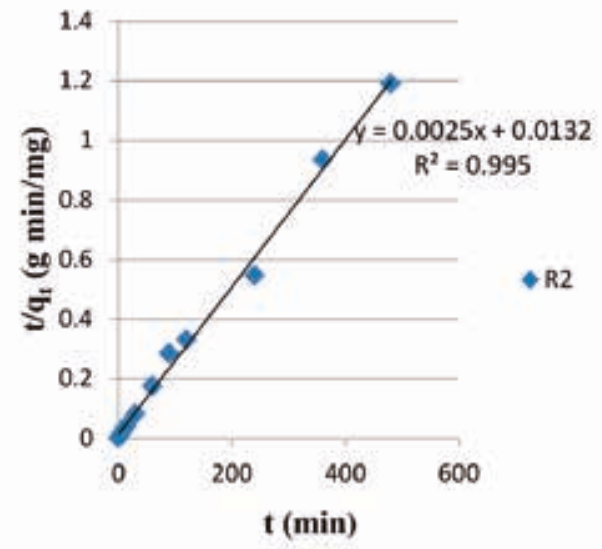

(a)

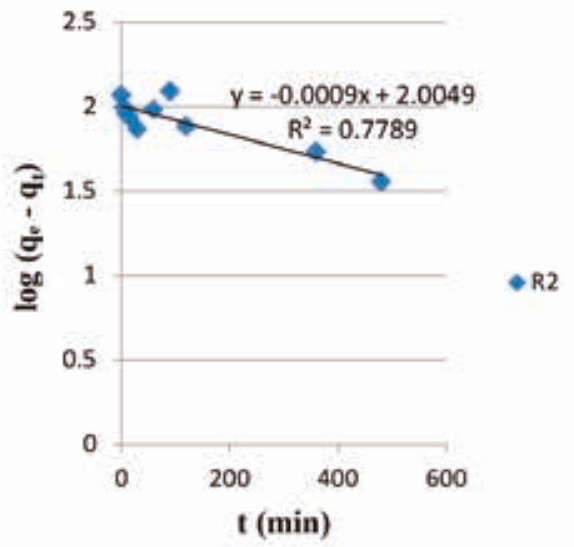

(b)

Figura 4. (a) Modelo cinético de pseudo-segundo orden y (b) Modelo cinético de pseudoprimer orden de la adsorción de Pb (II) sobre PTPE (R2).

Así también, en la tabla 3 se presentan los parámetros obtenidos del ajuste de los datos experimentales de la adsorción de Pb (II) sobre PTPE con el modelo cinético de pseudosegundo orden y con el modelo cinético de pseudo-primer orden. 
Tabla 3. Parámetros de los modelos cinéticos de pseudo-segundo orden y pseudo-primer orden obtenidos de la adsorción de $\mathrm{Pb}$ (II) sobre PTPE.

\begin{tabular}{ccccc}
\hline Muestra & $\mathrm{R} 1$ & $\mathrm{R} 2$ & $\mathrm{R} 3$ & Promedio \\
\hline Pseudo 2do. orden & & & & \\
\hline $\mathrm{K}_{2} \mathrm{~g} /(\mathrm{mg} \cdot \mathrm{min})$ & 0,0001685 & 0,0004735 & 0,0003079 & 0,0003166 \\
$\mathrm{q}_{\mathrm{e} \text { (cal) }}(\mathrm{mg} / \mathrm{g})$ & 400 & 400 & 400 & 400 \\
$\mathrm{q}_{\mathrm{e}(\mathrm{exp})}(\mathrm{mg} / \mathrm{g})$ & 449,07 & 439,2 & 417,45 & 435,24 \\
$\mathrm{R}^{2}$ & 0,9631 & 0,995 & 0,9915 & 0,98 \\
\hline Pseudo $1 \mathrm{er}$ orden & & & & \\
\hline $\mathrm{K}_{1}(\mathrm{~L} / \mathrm{min})$ & 0,0027636 & 0,0020727 & 0,0469812 & 0,017273 \\
$\mathrm{q}_{\mathrm{e}(\mathrm{cal})}(\mathrm{mg} / \mathrm{g})$ & 159,184 & 101,135 & 1068,809 & 443,043 \\
$\mathrm{q}_{\mathrm{e}(\exp )}(\mathrm{mg} / \mathrm{g})$ & 449,07 & 439,2 & 417,45 & 435,24 \\
$\mathrm{R}^{2}$ & 0,3537 & 0,7789 & 0,5352 & 0,56 \\
\hline
\end{tabular}

\section{CONCLUSIONES}

De los resultados obtenidos se concluye que los residuos de pelos del proceso de pelambre enzimático, tratados por hidrólisis ácida, son bioadsorbentes eficaces con una capacidad máxima de adsorción de 321,2 mg Pb (II)/g pelo. Asimismo, se determinó que la adsorción de $\mathrm{Pb}$ (II) es dependiente del $\mathrm{pH}$, siendo el $\mathrm{pH}$ óptimo de 7 y se estableció que el comportamiento de adsorción de $\mathrm{Pb}$ (II) sobre residuos de pelos tratados del pelambre enzimático es descrito de forma precisa por el modelo de la isoterma de Langmuir, obteniéndose una capacidad máxima de adsorción superior a otros bioadsorbentes. Además, se determinó que el modelo cinético de pseudo-segundo orden es el que mejor se ajusta a los datos experimentales obtenidos, siendo la adsorción química el paso limitante de la velocidad de adsorción.

\section{AGRADECIMIENTOS}

Se agradece al Fondo de Investigación y Desarrollo para la Competitividad (FIDECOM) que a través de los Proyectos de Innovación Productiva para Empresas Individuales (Convenio No022-FINCYT-FIDECOM-PIPEI-2012), a la empresa HELIANTHUS S.A.C y al Centro de Investigación en Química, Toxicología y Biotecnología Ambiental (CIQTOBIA) del Departamento Académico de Química de la UNALM, se logró realizar esta investigación. 


\section{REFERENCIAS BIBLIOGRÁFICAS}

1. García MM, Zárate MA, Rojas CI. Guía técnica para la minimización de residuos en curtiembres. Lima: CEPIS; 1993.

2. CPTS (Centro de Promoción de Tecnologías Sostenibles). Guía técnica de producción más limpia para curtiembres. La Paz: Centro de Promoción de Tecnologías Sostenibles; 2003.

3. García Roig M, Ramírez PFI, Manzano MT. Bioadsorción de metales pesados de aguas ácidas de minas (y II) sobre residuos con queratina (pelos de cerdo, pluma de ave) y quitina (caparazones de crustáceos). Ing Quím. 2004; 184 - 204.

4. Hawkins TR. Remoción de cromo total de efluentes de la industria de galvanoplastia por adsorción con gel SOW-Fe. [Tesis para optar el título de Ingeniero Ambiental]. Lima: Universidad Nacional Agraria La Molina. Perú; 2012.

5. Ghosh A, Collie SR. Keratinous Materials as Novel Absorbent Systems for Toxic Pollutants. Def Sci J. 2014; 64(3): 209-221.

6. Zhang $\mathrm{H}$. Biosorption of heavy metals from aqueous solutions using keratin biomaterials. [Tesis doctoral]. Barcelona: Universitat Autónoma de Barcelona; 2014.

7. Larenas UC, Andrango D, Inga P. Estudio isotérmico de biosorción de plomo en aguas utilizando residuos vegetales. La Granja. 2008; 8(2): 3-8.

8. García Roig M, Ramírez PFI, Manzano MT. Bioadsorción de metales pesados de aguas ácidas de minas (I) sobre residuos de levaduras cerveceras. Ing Quím. 2003; 400: 155 168.

9. Goel, J.; Kadirvelu, K.; Rajagopal, Ch.; Garg, V. K. Removal of lead (II) by adsorption using treated granular activated carbon: Batch and column studies. J Hazard Mater. 2005; B125: 211-220.

10. Hidalgo VAR. Biosorción de plomo y cadmio mediante el aprovechamiento de residuos de madera (aserrín de pino) y extractos de algas marinas (alginato de calcio). [Tesis para obtener el título profesional de Biólogo]. Michoacán: Universidad Michoacana de San Nicolás de Hidalgo; 2010.

11. Lara M. Caracterización y aplicación de biomasa residual a la eliminación de metales pesados. [Tesis doctoral]. Granada: Universidad de Granada; 2008.

12. Zahra N. Lead Removal fromWater by Low Cost Adsorbents: A Review. Pak J Anal Environ Chem. 2012; 13(1): 01-08.

13. Bernard E, Jimoh A, Odigure JO. Heavy Metals Removal from Industrial Wastewater by Activated Carbon prepared from Coconut Shell. Res J Chem Sci. 2013; 3(8): 3-9.

14. Sekimoto Y, Okiharu T, Nakajima H, Fujii T, Shirai K, Moriwaki H. Removal of Pb (II) from water using keratin colloidal solution obtained from wool. Environ Sci Pollut Res. 2013; 20(9):6531-8. 
15. Inga MPA. Estudio isotérmico de biosorción de cadmio, cromo, plomo y zinc en solución acuosa empleando el pinzote (raquis) de plátano (Musa paradisiaca L.). [Tesis para obtener el título en Ingeniería en Biotecnología de los recursos naturales]. Cuenca: Universidad Politécnica Salesiana; 2012.

16. Muñoz CJC. Biosorción de plomo (II) por cáscara de naranja "Citrus cinensis" pretratada. [Tesis para obtener el título profesional de Químico]. Lima: Universidad Nacional Mayor de San Marcos; 2007.

17. Romero GJ, Parra VF, Cano RI, Rodríguez E, Ríos AJ, Fuentes HR, et al. Biosorción de $\mathrm{Pb}$ (II) por biomasa de Agave tequilana Weber (Agave azul). Rev Mex Ing Quím. 2007; 6(3): 295-300. 